\title{
Title: The evolution of social monogamy in mammals
}

\author{
Authors: D. Lukas ${ }^{1 *}$, T. H. Clutton-Brock ${ }^{1}$
}

\author{
Affiliations: \\ $5 \quad{ }^{1}$ Department of Zoology \\ University of Cambridge \\ Downing Street \\ Cambridge CB2 3EJ \\ U.K. \\ 10 \\ *Correspondence to: d1384@cam.ac.uk
}

\begin{abstract}
:
15 The evolution of social monogamy has intrigued biologists for over a century. One possible explanation is that social monogamy has developed where there is strong selection for some form of paternal care. Here, we show that the ancestral condition for all non-human mammalian groups is of solitary females with males occupying separate but overlapping ranges; that the evolution of social monogamy has not been associated with a high risk of male infanticide and

20 that paternal care is a consequence rather than a cause of social monogamy. In mammals, social monogamy appears to have evolved where breeding females are intolerant of each other and female density is low, suggesting that it has evolved as a mating strategy where males are unable to defend access to multiple females.
\end{abstract}

One Sentence Summary: Social monogamy has evolved in solitary mammals as a male mating strategy and is not a consequence of selection for paternal care. 


\section{Main Text:}

Despite extensive interest in the evolution of social monogamy stimulated by its prevalence in humans (1-3), its distribution in non-human mammals continues to puzzle evolutionary biologists (4). In contrast to birds, social monogamy in mammals is closely associated with genetic monogamy and the incidence of extra-pair mating is generally low in socially monogamous societies (5-7). The two main explanations are that it is a consequence of selection for some form of paternal care, such as contributions to carrying or provisioning young or their protection from infanticide by competing males (8); or that social monogamy represents a male guarding strategy and has evolved where males are unable to defend access to more than one female $(9,10)$, either because of mutual intolerance of breeding females $(11,12)$ or because large female home-ranges prevent effective defence by males of territories encompassing the

40 ranges of more than one female $(13,14)$. A recent comparative analysis (15) using a Bayesian approach identified six evolutionary transitions to monogamy in primates and concluded that social monogamy is derived from an ancestral condition where both sexes are social and live in unstable groups, supporting the suggestion that its evolution may be associated with the risk of male infanticide. Here, we identify sixty separate evolutionary transitions to social monogamy in

45 mammals, assess the characteristics of the species in which they occurred and use them to test the predictions of alternative explanations of the evolution of social monogamy.

We initially classified the social systems of all mammalian species for which information was available $(n=2543)$ as either solitary (females live solitary in separate home-ranges and encounter males only during mating), socially monogamous (a single breeding female and a 50 single breeding male associate with each other for more than one breeding season, with or 
without nonbreeding offspring) or group-living (where females forage or sleep in groups). Group-living species include those where groups of breeding females are unstable, as in the case of ungulate herds or the roosting groups of some bats, as well as species where several breeding females associate with each other for more than one breeding season in stable groups, whether or

55 not they always forage together (as in many social carnivores and primates)(Supplementary Data). Species were classified as showing paternal care if males regularly contribute to feeding or carrying offspring $(2,16)$. After reconstructing the most parsimonious sequence of transitions across a recently derived mammalian supertree $(17,18)$, all inferences were confirmed using likelihood-based reconstruction approaches $(19,20)$. We first tested for associations between the

60 distribution of social monogamy and several social and ecological traits using non-parametric tests, MCMCglmm (21), and phylogenetic independent contrasts $(22,23)$. Next, we assessed the importance of any associated factors in predicting transitions to social monogamy by comparing inference models in Bayestraits' Discrete (23, 24).

65 The distribution of social monogamy

Of the 2543 mammalian species whose social systems could be classified, females were classified as solitary in 1740 species (68\%), as socially monogamous in 226 species (9\%), as living in unstable groups in 133 species (5\%), and as living in stable groups in a further 444 species $(17 \%)$. The proportion of socially monogamous species in our sample is slightly higher

70 than frequently reported earlier estimates $(3 \%,(1))$, but is still an order of magnitude lower than in birds, where $90 \%$ of species are considered to be socially monogamous (25). Social monogamy occurs more frequently in some mammalian Orders, such as Primates (104 of 359 species, 29\%) and Carnivora (33 of 201 species, 16\%), and is uncommon in others, such as 
Rodentia (57 of 951 species, 6\%), and absent in a few, including Cetacea ( 0 of 54 species) (see 75 Table S1).

\section{$\underline{\text { Transitions to social monogamy }}$}

Our phylogenetic reconstruction shows that, in the common ancestor of all mammalian species, females were solitary and males occupied ranges or territories overlapping several

80 females and that solitary living is the ancestral condition for the ancestors of all mammalian Orders (see Figure 1), with the possible exception of elephant shrews (Macroscelidea) and hyraxes (Hyracoidea). All approaches to reconstructing evolutionary sequences support this inference for the 2288 species included in the updated mammalian supertree and the likelihood that the common ancestor was solitary is 0.99 for all approaches. Closely related species

85 generally have the same breeding system and female sociality has a strong phylogenetic signal: maximum likelihood estimate of Pagel's lambda was 0.93 for solitary living, 0.92 for social monogamy, and 0.86 for group living; all lambda estimates were significantly different from 0 (no phylogenetic signal) based on likelihood ratio tests. Similarly, the phylogenetic signal for all three social systems combined using Blomberg's K was significantly different from a chance 90 distribution of sociality across species $(0.20, Z=-10.99, p=0.001)$.

Parsimonious reconstructions suggest that 60 separate transitions to social monogamy from solitary ancestors are necessary to explain the distribution of social monogamy among current species. All socially monogamous species in our dataset are derived from an ancestor where females were solitary and lived in individual home-ranges and males ranged

95 independently. No transitions to social monogamy from an ancestor where females lived in groups, either in primates or other mammals, were detected. The results of Bayestraits' Discrete 
analyses performed for each Order in which socially monogamous species occur confirmed that social monogamy is always derived from ancestors where females are solitary. Models in which transition rates to social monogamy were forced to occur equally from group-living ancestors 100 and solitary ancestors performed significantly worse than models in which all socially monogamous species descend from a solitary ancestor (Likelihood ratio test (lrt) all $\mathrm{p}<0.005$; Table S2). We detected group-living in sister taxa of socially monogamous species in at least three instances (banded mongooses, Mungos mungo; bamboo lemur, Hapalemur griseus; and Goeldii's monkey, Callimico goeldii), all of which probably represent secondary transitions to 105 group living from a socially monogamous ancestor.

\section{$\underline{\text { Social monogamy and male care }}$}

Regular provisioning or carrying of young by males has been recorded in 127 (56\%) of the 226 socially monogamous mammals, and in many socially monogamous species males

110 neither feed or carry young. While it is often difficult to exclude the possibility of any form of male contribution to the care of young, detailed field studies have found no evidence of any form of male contribution in several of the 99 species where males neither carry nor provision young. For example, in dik dik, where males are both genetically and socially monogamous and are closely associated with their mates, they provide no contributions to guarding, carrying, feeding

115 or teaching young or to any other obvious form of paternal care (26). The distribution of paternal care in contemporary socially monogamous species is closely associated with the form and distribution of maternal care: where females carry and/or provision offspring, males commonly contribute to the same activities. Paternal care in non-monogamous species is found only in species that derive from a socially monogamous ancestor, such as Mungos mungo (27), 
Comparisons suggest that paternal care probably contributes to the fitness of both sexes: females in socially monogamous species with bi-parental care produce more litters per year (median 2, range $0.9-9, \mathrm{n}=48$ species) than socially monogamous species without bi-parental care (median 1, range $0.2-7, \mathrm{n}=37$ species; $\mathrm{F}=4.43, \mathrm{p}=0.04$, phylogenetic Anova (phyA) $\mathrm{p}=0.32$ )

125 or than solitary species (median 1.1 , range $0.2-7, n=242$ species; $F=7.56, p=0.006$, phyA $p=0.27$ ). Increases in the reproductive rates of females probably have benefits to males, for males sire offspring in more breeding cycles in socially monogamous species with paternal care (median 6 breeding seasons, range $4.5-8$ breeding seasons, $\mathrm{n}=11$ species) compared to socially monogamous species where males do not provide care (median 3 breeding seasons, range 2-8

130 breeding seasons, $\mathrm{n}=8$ species; $\mathrm{F}=4.98, \mathrm{p}=0.04$; phyA $\mathrm{p}=0.21$ ), even though there are no differences in male tenure length (with paternal care median 47 months, without median 45 months; $\mathrm{F}=2.10, \mathrm{p}=0.17$, phyA $\mathrm{p}=0.35$ ).

Although paternal care and social monogamy are associated, analysis of transitions suggests that male care is probably a consequence rather than a cause of the evolution of social

135 monogamy. Approximately half of all independent transitions to paternal care have occurred in instances where social monogamy was already established while, in the other cases, the evolution of paternal care occurred at the same time as a transition to social monogamy. Inferences from Bayestraits models indicate that paternal care is a secondary adaptation, as transitions to social monogamy are inferred to occur first on branches where both traits evolved $140 \quad(\operatorname{lrt} \mathrm{p}=0.002 ;$ Table S2).

$\underline{\text { Social monogamy and male infanticide }}$ 
An alternative suggestion is that social monogamy allows males to protect their offspring from attacks by infanticidal competitors and has evolved for this reason (30). However, the

145 available evidence suggests that male infanticide is unlikely to be the principal mechanism for the evolution of social monogamy in mammals. Male infanticide is typically found in species where the duration of lactation exceeds the duration of gestation $(8,31)$ but this is the case in relatively few socially monogamous species (20 of 75 species, $27 \%$ ) compared to species where females are solitary (148 of 335 species, 44\%; W=11733, $\mathrm{p}=0.34$; phylogenetic independent

150 contrasts (pic) $\mathrm{t}=-1.63, \mathrm{p}=0.10$ ) and Bayestraits models also provide no evidence of an association between the evolution of social monogamy and lactation durations that exceed gestation (lrt $\mathrm{p}>0.40$, Table S2). Although the prevalence of male infanticide is lower among socially monogamous species (4 of 45 species, 9\%) than among solitary species (24 of 88 species, $27 \% ; \mathrm{W}=1542.5, \mathrm{p}=0.01$ ), this difference does not appear to be a consequence of a direct

155 association between social monogamy and male infanticide, as analysis of phylogenetic independent contrasts $(\mathrm{t}=-0.402, \mathrm{p}=0.69)$ and Bayestraits models suggest an independent evolution of the two traits (lrt $\mathrm{p}>0.90$, Table S2).

Social monogamy and the ecological defensibility of females

160 The main alternative explanation of the distribution of social monogamy in mammals is that it has evolved where females are solitary and males are unable to defend access to more than one female at a time (9). Evidence that socially monogamous species are derived from ancestors where females are solitary (see above) supports this suggestion. Moreover, unlike previous analyses $(2,4)$, our data show that socially monogamous mammals live at significantly lower 165 densities (median 15 individuals per square kilometre, $\mathrm{n}=89$ species) than solitary species 
(median 156 individuals per square kilometre, $\mathrm{n}=411$ species; $\mathrm{W}=10746.5, \mathrm{p}<0.001$; phylogenetically controlled binomal GLM in MCMCglmm (pMCMC) $\mathrm{p}=0.007$ )(Figure 2). Socially monogamous species have, on average, higher body mass (average 966g, median 873) compared to solitary species (average 579g, median 308g; $\mathrm{W}=40733$, $\mathrm{p}=0.001 ; \mathrm{pMCMC}=0.34$ ),

170 which may contribute to their low density. However, the residuals of a phylogenetic controlled regression of population density on body mass are significantly lower for socially monogamous species than for solitary species $(\mathrm{W}=10421, \mathrm{p}<0.001$; $\mathrm{pMCMC}<0.001)$, indicating that size differences alone do not account for the low density of socially monogamous species and that some other factor, such as dependence on low density food supplies may be important.

175 Despite the association between social monogamy and low population density, there is no significant difference in female home-range size between socially monogamous (median 0.21 square kilometres, $n=71$ species) and solitary species (median 0.53 square kilometres, $n=185$ species; $\mathrm{W}=5553, \mathrm{p}=0.06 ; \mathrm{pMCMC}=0.11$ ), even when differences in body mass are controlled for $(\mathrm{W}=6100, \mathrm{p}=0.70 ; \mathrm{pMCMC}=0.08)$. This suggests that there may be greater overlap of home ranges between females in solitary species than in socially monogamous ones and comparative data for primates (the only taxonomic group for which comparative data are available) supports this conclusion: in a sample of 26 socially monogamous primates, home-ranges overlap on average by $21 \%$ (median 17\%), whereas, in species where females are solitary, the ranges of females overlap on average by $49 \%$ (median $58 \%, \mathrm{n}=5$ species; $\mathrm{F}=7.08, \mathrm{p}=0.01$; phyA $\mathrm{p}=0.28$ ).

The relatively high incidence of social monogamy in Primates and Carnivora compared to more herbivorous Orders (including Rodentia and Ungulates) suggest that there may be an association between the evolution of social monogamy and reliance on resources of high nutritional qualities but low abundance. A similar association between social monogamy and low 
density resources occurs within Orders. For example, in $90 \%$ ( 79 of 87 ) of socially monogamous

190 primates, fruit constitute the main part of the diet, whereas fruit is the single most important food for only $28 \%$ ( 13 of 46 ) of solitary primate species ( $\mathrm{W}=749.5, \mathrm{p}<0.001$; pic $\mathrm{t}=0.68, \mathrm{p}=0.50)$. In contrast, foods of low nutritional value (gum, bark, fungi) are included in the diet of significantly more solitary (36 of $46,78 \%$ ) than socially monogamous primate species ( 35 of $87,40 \%$; $\mathrm{W}=3066.5, \mathrm{p}<0.001 ;$ pic $\mathrm{t}=-3.67, \mathrm{p}<0.001)$.

195 Analyses of patterns of sexual dimorphism also suggest that competition between females may be more intense in socially monogamous species than in solitary ones. While males are heavier than females in 134 of 170 species (79\%) where females are solitary, male-biased sexual dimorphism is found in only 21 of 44 socially monogamous species $(48 \%$; $\mathrm{W}=2736.5, \mathrm{p}<0.001$; pic $\mathrm{t}=1.53, \mathrm{p}=0.13)$. This difference does not appear to be a consequence of a reduction in

200 dimorphism after the transition to social monogamy, for the sequence of transitions as inferred by the most likely Bayestraits models suggests that social monogamy only evolved in species in which females are at least as large as males (lrt $\mathrm{p}<0.05$; Table S2).

\section{Discussion}

Like previous analyses $(2,15)$ our results suggest that the evolution of monogamy has been restricted to particular ancestral states. However, our conclusion that social monogamy is always derived from an ancestral state in which females forage alone and live in separate homeranges and male ranges overlap those of several females contrasts with recent suggestions that, in primates, it is derived from ancestors in which females and males live in unstable groups (15).

210 This difference is unlikely to be a consequence of contrasts between primates and other mammals, for all transitions to social monogamy among primates in our dataset were also from 
ancestors where females live in separate home ranges. Instead, it may be a consequence of a contrast in the classification of breeding systems: Shultz et al. classify socially monogamous species that are accompanied by non-breeding offspring (singular breeders) as group-living and

215 do not distinguish between them and plural breeders where groups include several breeding females. As a result, some species that we classify as socially monogamous were classified by Shultz et al. as group-living.

The association between social monogamy and low population density also differs from previous analyses which found no significant difference in population density between socially monogamous species and those where females live in separate home ranges $(2,4)$. In this case, it seems likely that the contrast is a result of differences in sample size between our analyses and previous analyses where sample size is less than 90 species. Our larger sample size also allowed us to assess whether changes in population density preceded transitions to social monogamy. Comparing average population density between solitary and socially monogamous species might

225 fail to detect a difference, because low population density does not necessarily lead to the evolution of social monogamy (18).

Our results consequently suggest that social monogamy evolved in mammals where competition between females was intense, breeding females were intolerant of each other and population density was low. Under these conditions, guarding individuals females may represent

230 the most efficient breeding strategy for males (9). In contrast to previous suggestions, the evolution of paternal care appears to succeed the evolution of social monogamy, suggesting that it is not a precondition for the evolution of social monogamy. In a small number of such singular cooperative breeders, secondary transitions have occurred to plural breeding by several females whose offspring are raised by all group members (e.g. banded mongooses, (32)). This suggests 
235 that there are two independent routes to female sociality in mammals. In species where females share part of their range with relatives, tolerance between breeding females led to the evolution of stable groups of breeding females and the development of different forms of polygyny (33, 34). An alternative route to sociality occurs in polytocous species when offspring are retained in the ranges of monogamous pairs and act as helpers for their parents (35). 


\section{References and Notes:}

1. D. Kleiman, Monogamy in mammals, Q. Rev. Biol. 39-69 (1977).

2. P. Brotherton, P. Komers, in Monogamy: mating strategies and partnerships in birds, humans and other mammals. Eds. C. Boesch, U. Reichard (Cambridge Univ. Press, Cambridge, 2003) pp. 42-58.

3. S. Gavrilets, Human origins and the transition from promiscuity to pair-bonding, Proc. Natl. Acad. Sci. U.S.A. 109, 9923-9928 (2012)

4. F. S. Dobson, B. M. Way, C. Baudoin, Spatial dynamics and the evolution of social monogamy in mammals, Behav. Ecol. 21, 747-752 (2010).

5. S. Griffith, I. Owens, K. Thuman, Extra pair paternity in birds: a review of interspecific variation and adaptive function, Mol. Ecol. 11, 2195-2212 (2008).

6. T. H. Clutton-Brock, K. Isvaran, Paternity loss in contrasting mammalian societies, Biol. Let. 2 , $513-6(2006)$.

255 7. A. Cohas, D. Allainé, Social structure influences extra-pair paternity in socially monogamous mammals., Biol. Let. 5, 313-6 (2009).

8. C. P. van Schaik in Primate males: causes and consequences of variation in group composition. ed. P. Kappeler (Cambridge Univ. Press, Cambridge, 2000) pp. 61-71.

9. S. Emlen, L. Oring, Ecology, Sexual Selection, and the Evolution of Mating Systems, Science 197, 215-224 (1977).

10. S. Shuster, M. Wade, Mating systems and mating strategies. (Princeton Univ. Press, Princeton, 2003).

11. J. Wittenberger, R. Tilson, The evolution of monogamy: hypotheses and evidence, Annu. Rev. 
Ecol. Syst., 197-232 (1980).

265 12. U. Reichard, in Monogamy: mating strategies and partnerships in birds, humans and other mammals. eds. C. Boesch, U. Reichard (Cambridge Univ. Press, Cambridge, 2003)

13. G. Orians, On the evolution of mating systems in birds and mammals, Am. Nat. 103, 589-603 (1969)

14. E. Geffen, D. MacDonald, Small size and monogamy: spatial organization of Blanford's 270 foxes, Vulpes cana, Anim. Behav. 44, 1123-1130 (1992)

15. S. Shultz, C. Opie, Q. D. Atkinson, Stepwise evolution of stable sociality in primates, Nature 479, 219-222 (2011).

16. C. Van Schaik, P. Kappeler, in Monogamy: mating strategies and partnerships in birds, humans and other mammals. eds. C. Boesch, U. Reichard (Cambridge Univ. Press, 275 Cambridge, 2003).

17. O. R. P. Bininda-Emonds et al., The delayed rise of present-day mammals, Nature 446, 50712 (2007).

18. S. A. Fritz, O. R. P. Bininda-Emonds, A. Purvis, Geographical variation in predictors of mammalian extinction risk: big is bad, but only in the tropics, Ecol. Letters 12, 538-49 $280 \quad$ (2009).

19. D. Schluter, T. Price, A. Mooers, D. Ludwig, Likelihood of ancestor states in adaptive radiation, Evolution 51, 1699-1711 (1997).

20. M. Pagel, Inferring the historical patterns of biological evolution, Nature 401, 877-84 (1999).

285 21. J. D. Hadfield, S. Nakagawa, General quantitative genetic methods for comparative biology: phylogenies, taxonomies and multi-trait models for continuous and categorical characters, $J$. 
Evol. Biol. 23, 494-508 (2010).

22. J. Felsenstein, Phylogenies and the comparative method, Am. Nat. 125, 1-15 (1985).

23. More detailed information on materials and methods is available on Science Online.

290 24. M. Pagel, A. Meade, Bayesian analysis of correlated evolution of discrete characters by reversible-jump Markov chain Monte Carlo, Am. Nat. 167, 808-825 (2006).

25. A. Cockburn, Prevalence of different modes of parental care in birds, Proc. R. Soc. London Ser. B 273, 1375-1383 (2006).

26. P. Brotherton, M. Manser, Female dispersion and the evolution of monogamy in the dik-dik, Anim. Behav, 54, 1413-1424 (1997)

27. M. Cant, Patterns of helping effort in co-operatively breeding banded mongooses (Mungos mungo), J. Zool. 259, 115-121 (2003).

28. P. C. Wright, Patterns of paternal care in primates, Int. J. Primat. 11, 89-102 (1990).

29. C. Schradin, G. Anzenberger, Infant carrying in family groups of Goeldi's monkeys

300 (Callimico goeldii), Am. J. Primat. 53, 57-67 (2001).

30. C. Van Schaik, R. Dunbar, The evolution of monogamy in large primates: a new hypothesis and some crucial tests, Behaviour 115, 30-62 (1990).

31. R. Palombit, in The Evolution of Primate Societies. eds. J. C. Mitani, J. Call, P. Kappeler, R. A. Palombit, J. B. Silk (Univ. of Chicago Press, Chicago, 2012) pp. 432-441.

305 32. M. Cant, Social control of reproduction in banded mongooses, Anim. Behav. 59, 147-158 (2000).

33. J. Hoogland, The black-tailed prairie dog: social life of a burrowing mammal (Univ. of Chicago Press, Chicago, 1995).

34. T. Clutton-Brock, Structure and function in mammalian societies, Phil. Trans. Royal Soc. B: 
Biol. Sciences 364, 3229-3242 (2009).

35. D. Lukas, T. Clutton-Brock, Cooperative breeding and monogamy in mammalian societies, Proc. R. Soc. London Ser. B 259, 2171-2176 (2012).

36. D. E. Wilson, D. M. Reeder, Mammal species of the world: a taxonomic and geographic reference (Johns Hopkins, Boston, 2005).

315 37. R. N. Nowak, Walker's mammals of the world. (John Hopkins, Baltimore, 1999).

38. P. E. Komers, P. N. Brotherton, Female space use is the best predictor of monogamy in mammals, Proc. R. Soc. London Ser. B 264, 1261-70 (1997).

39. M. van Noordwijk, C. Van Schaik, in Infanticide by males and its implications, C. van Schaik, C. H. Janson, Eds. (Cambridge University Press, Cambridge, 2000), pp. 322-360.

40. D. T. Blumstein, in Infanticide by males and its implications eds. C. van Schaik, C. Janson, (Cambridge Univ. Press, Cambridge, 2000) pp. 178-197.

41. S. K. M. Ernest, Life history characteristics of placental nonvolant mammals, Ecology 84, 3402 (2003).

42. K. E. Jones et al., PanTHERIA: a species-level database of life history, ecology, and geography of extant and recently extinct mammals, Ecology 90, 2648-2648 (2009).

43. J. P. de Magalhães, J. Costa, A database of vertebrate longevity records and their relation to other life-history traits., J. Evol. Biol. 22, 1770-4 (2009).

44. F. Pearce, C. Carbone, G. Cowlishaw, N. J. Isaac, N. J. Space-use scaling and home range overlap in primates. Proc. R. Soc. London Ser. B 280, 1751 (2013).

330 45. J.M. Gómez, M. Verdú, Mutualism with plants drives primate diversification. Syst. Biol. 61, 567-577 (2012).

46. D. Lukas, T. H. Clutton-Brock, Group structure, kinship, inbreeding risk and habitual female 
dispersal in plural-breeding mammals. J. Evol. Biol. 24, 2624-2630 (2011).

47. P. Jarman, Mating system and sexual dimorphism in large terrestrial, mammalian herbivores. Biol. Rev. 58, 485-520 (1983).

48. A. Loison, J. M. Gaillard, C. Pelabon, N. G. Yoccoz, What factors shape sexual size dimorphism in ungulates? Evol. Ecol. Res. 1, 611-633 (1999).

49. R. J. Smith, J. M. Cheverud, Scaling of sexual dimorphism in body mass: a phylogenetic analysis of Rensch's rule in primates. Int. J. Primatol. 23, 1095-1135. (2002).

340 50. J. L. Isaac, Potential causes and life-history consequences of sexual size dimorphism in mammals. Mam. Rev. 35, 101-115 (2005).

51. E. Paradis, J. Claude, K. Strimmer, APE: analyses of phylogenetics and evolution in R language. Bioinf. 20, 289-290 (2004).

52. R Development Core Team, $R$ : A Language and Environment for Statistical Computing. (R

345 Foundation for Statistical Computing, Vienna, 2010).

53. W. P. Maddison, D.R. Maddison, Mesquite: a modular system for evolutionary analysis. Version $2.75 \mathrm{http}: / /$ mesquiteproject.org (2011).

54. L. J. Harmon, J. T. Weir, C. D. Brock, R. E. Glor, W. Challenger, W. GEIGER: investigating evolutionary radiations. Bioinf. 24, 129-131 (2008).

350 55. S. W. Kembel, P. D. Cowan, M. R. Helmus, W. K. Cornwell, H. Morlon, D. D. Ackerly, S. P. Blomberg, C. O. Webb, Picante: R tools for integrating phylogenies and ecology. Bioinf. 26, 1463-1464 (2010).

56. A. Grafen, The phylogenetic regression. Phil. Trans. Royal Soc. B: Biol. Sciences 326, 119157 (1989).

355 57. J. Hadfield, MCMCglmm: Markov chain Monte Carlo methods for Generalised Linear 
Mixed Models. Retrieved from:

cran.uvigo.es/web/packages/MCMCglmm/vignettes/Tutorial.pdf (2010).

58. M. Plummer, N. Best, K. Cowles, K. Vines, Convergence Diagnosis and Output Analysis for MCMC. R News, 6, 7-11 (2006).

360 59. A. Gelman, D. Rubin, D. Inference from iterative simulation using multiple sequences. Stat. Science, 7, 457-511 (1992).

60. C. D. L. Orme, R. P. Freckleton, G. H. Thomas, T. Petzoldt, S. A. Fritz, N. J. Isaac, N. J. B. caper: Comparative Analyses of Phylogenetics and Evolution in R, Methods Ecol. Evol. (in press).

365

370

\section{Acknowledgments:}

We thank Steve Dobson for detailed comments, D. Blumstein and N. Solomon for critical feedback on the classification of species, and everyone in the Large Animal Research Group in Cambridge for helpful discussions. Data is available as Supplementary Material. The European 375 Research Council provided the funding for this study through a grant to T.H.C.B. 
Fig. 1. Evolutionary pathway to monogamy and singular cooperative breeding in mammals

In mammals, social monogamy derives directly from ancestral social systems in which females live in separate home-ranges and male ranges overlap those of several females. Social 380 monogamy appears to have evolved in species where females rely on high quality, low density diets and breeding females are intolerant of each other and female density is low, preventing breeding males from guarding more than one breeding female. In some monogamous lineages where females are polytocous and habitats are unpredictable, systems where one female monopolizes breeding and her young are raised by other group members have evolved. Social 385 groups of this kind are distinct from groups that include multiple breeding females which appear to have evolved directly from solitary ancestors in lineages where direct competition for resources is lower, female density is higher, and breeding females tolerate each others' presence in the same group.

390 Picture credit: All pictures under Creative Commons Attribution License: http://en.wikipedia.org/wiki/File:Eichhörnchen_Düsseldorf_Hofgarten_Crisco_edit.jpg http://en.wikipedia.org/wiki/File:Nine-banded_Armadillo.jpg

http://en.wikipedia.org/wiki/File:Cheetah_Feb09_02.jpg http://en.wikipedia.org/wiki/File:Numbat.jpg

395 https://commons.wikimedia.org/wiki/File:Dik-dik_\%28male\%29_-Tarangire_National_Park_Tanzania.jpg https://commons.wikimedia.org/wiki/File:Panamanian_Night_Monkeys2.jpg https://commons.wikimedia.org/wiki/File:Rhynchocyon_petersi_from_side.jpg https://commons.wikimedia.org/wiki/File:Aonyx_cinera_in_Zoo-002.jpg http://www.flickr.com/photos/bobowen/4252523174/

400 http://www.flickr.com/photos/finchlake/5460526644/in/photostream/ http://www.flickr.com/photos/digitalart/2267158454/

http://www.flickr.com/photos/jeremyweber/7744688998/sizes/z/in/photostream/ 
Fig 1.

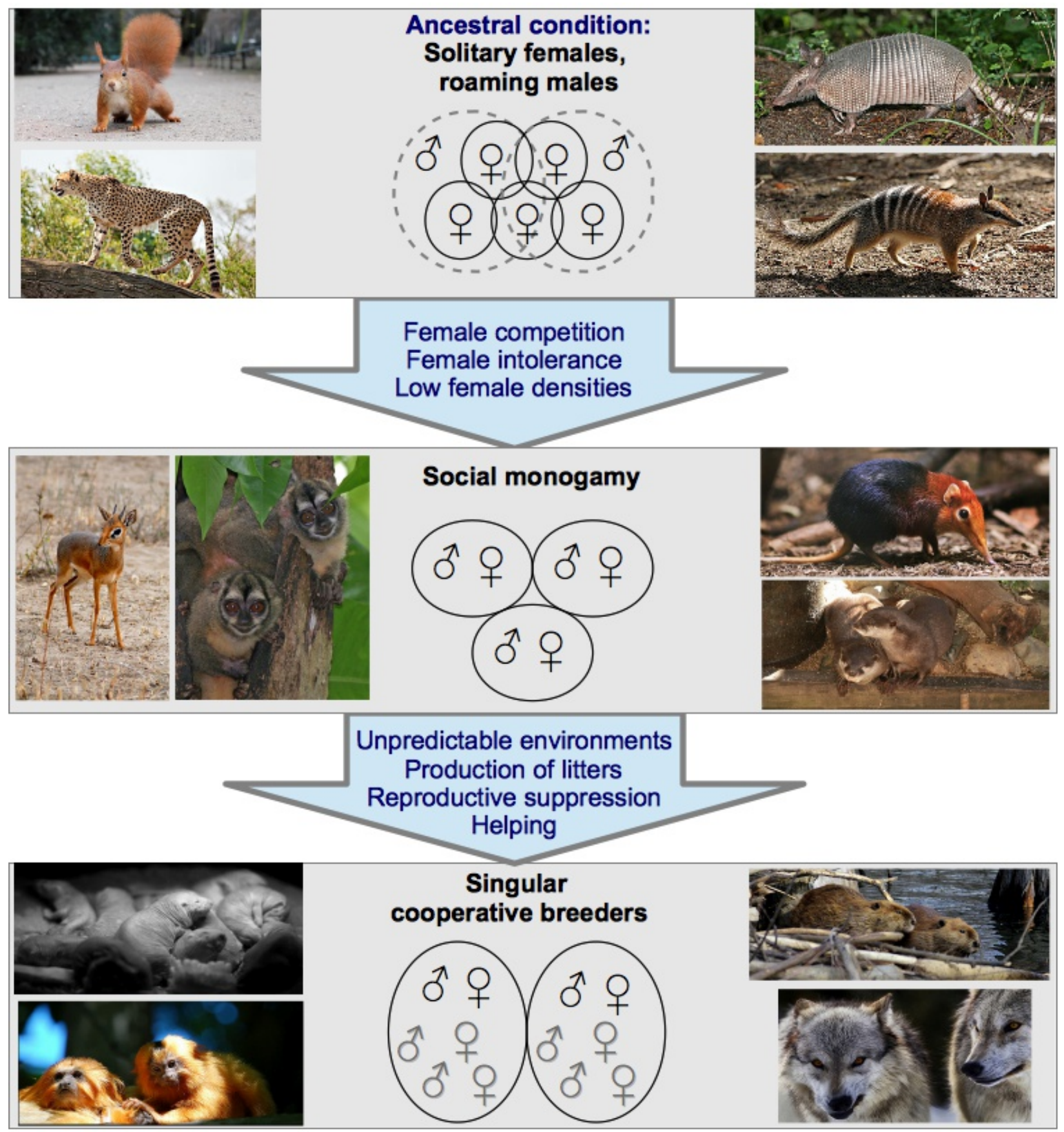


Fig. 2. Fitted values of the probability that a species is socially monogamous given a population density obtained by a binomial GLM (dashed line).

The blue dots are the observed values for solitary species $(n=411)$, the red crosses the observed

410 values for socially monogamous species ( $\mathrm{n}=89,18 \%$ of all species), values can overlap (e.g. there are four socially monogamous species with a log population density of -2). Population density (logarithm of the number of individuals per $\mathrm{km}^{2}$ ) has a significant influence on the probability of that a species is socially monogamous or solitary. At the highest population densities, there is only a $6 \%$ probability that a species will be socially monogamous, whereas the 415 probability rises to $44 \%$ at the lowest population densities. Several of the socially monogamous species showing high population densities are cooperative breeders, where many of the adult individuals do not breed. 
Fig 2.

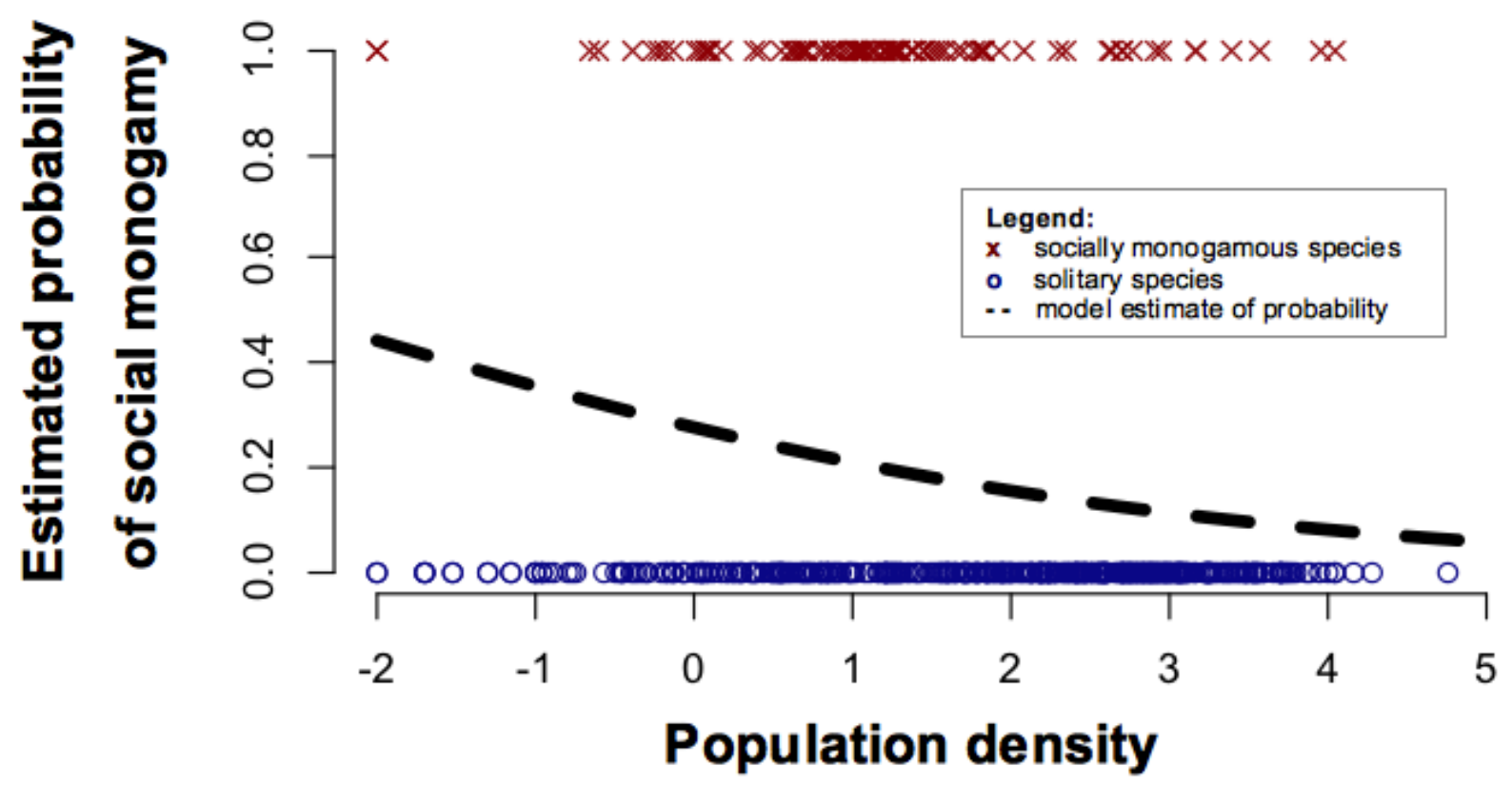

(log individuals per $\mathrm{km}^{2}$ ) 


\section{Supplementary Materials:}

Materials and Methods

430 Tables S1-S2

References (36-60)

Dataset

\section{Material and Methods}

\section{$\underline{\text { Classification of social system }}$}

Information on the social system and the occurrence of paternal care was collected during a systematic literature review where we searched for information on every non-human mammalian species (following the nomenclature of (36)), collecting information from the primary literature, by searching for each species latin name on Google Scholar, from encyclopedias (37), and from published reviews (including 1,38). We classified a species as

445 socially monogamous if the majority of breeding females $(>50 \%)$ share a home-range throughout the year with one male, but no other conspecific breeders. In most cases, socially monogamous pairs exhibit stable relationships that endure for at least two breeding seasons. Species were classified as solitary if females do not tolerate conspecifics of either sex for protracted periods within their home-range. Species were classified as group-living if two or more breeding females associate within the same home-range or at the same breeding spot. Species were classified as showing paternal care if males regularly contribute to feeding or carrying offspring $(2,16)$. Information on the occurrence of infanticide was compiled from previous reviews $(39,40)$. 


\section{Data on ecological and life-history parameters}

We compiled information on the potential correlates of social monogamy from the following reviews and databases: body mass, litters per year, and length of gestation and lactation (41-43); population density and home-range size (42); home-range overlap (44); diet (45); male tenure length (46); and sexual dimorphism (47-50). All continuous variables were logtransformed before analyses.

The updated mammalian supertree (18) was used as the basis for the phylogenetic analyses. The tree was truncated to match the species in the dataset using functions of the package 'ape' (51) in the statistical software $\mathrm{R}$ (52). We resolved polytomies randomly for all analyses that require bifurcating trees, and repeated each analysis with three independent resolutions, which in all cases gave consistent results.

A variety of approaches were used to reconstruct ancestral states for all mammalian species and for each mammalian Order. We inferred ancestral states using the multistate reconstruction in Bayestraits' Discrete and the maximum likelihood approach for discrete characters implemented in the package 'ape' in $\mathrm{R}$ and in Mesquite (53). The reconstructed states were used to determine the ancestral social system before transitions to social monogamy. We estimated Pagel's lambda as a measure of phylogenetic signal for each social system using functions in the R-package 'geiger' (54), and calculated Blomberg's K for all three social systems combined (socially monogamous $=0$, solitary $=1$, group living $=2$ ) using functions in the Rpackage 'picante' (55).

We subsequently tested whether transitions to social monogamy were constrained to 475 solitary species using the module "Discrete" (24) from the program "Bayestraits" (20). In all 
Bayestraits analyses, likelihoods of independent and dependent models of trait evolution were estimated based on the dated phylogeny, a phylogeny with branch-lengths transformed according to Grafen (56), and a phylogeny with all branch lengths set to equal one. Analyses based on trees in which all branch length were set to be equal to one produced the best likelihoods, and we 480 present their results below. We allowed the traits to vary their rate of evolution across the tree, and run each analysis for 50 maximum likelihood estimations. We assessed significance between dependent and independent models by comparing the likelihood ratio statistic against a chisquared distribution with four degrees of freedom (24). If results suggested that transition in one of the variables depended on the state of the other variable (e.g. transition to social monogamy 485 only occurring from solitary ancestors), we repeated the analysis constraining the rate of transition in the dependent variable to be equal for both states of the other variable. Unconstrained models were considered to explain the data significantly better if the likelihood ratio statistic exceeded a chi-squared distribution with one degree of freedom.

We performed a series of different statistical analyses depending on the hypotheses and

490 the type of data. When assessing the relationship between social monogamy and other binary traits, such as paternal care, we first compared the occurrence of the trait between solitary and socially monogamous species using Wilcoxon-tests and we regressed phylogenetic independent contrasts. Next, we assessed whether the two traits evolved independently through model comparisons in Bayestraits' Discrete. For tests where we predicted that social monogamy or 495 paternal care might influence a continuous response variable, such a the number of litters per year, we used Anova and phylogenetic Anova in the R-package 'geiger'. In cases where we predicted that a continuous variable, such as population density, might influence the occurrence of social monogamy, we performed Wilcoxon-tests and binomial GLMMs. Regressions were 
performed using MCMCglmm (21), including the phylogenetic relationship betweens species as

500 covariance matrix. In MCMCglmm, terms were considered statistically significant when the calculated $\mathrm{pMCMC}$ values were less than 0.05 . We ran analyses initially with two different priors, either fixing the variances at 1 and covariances at 0 , or using a very informative prior (57). The two different priors led to highly similar estimates for the fixed terms, and we report the values from the fixed prior. Each analysis was repeated three times, and was checked for convergence using the Gelman-Rubin statistic to compare within- and between- chain variance in the R-package 'coda' (58). In all cases the potential scale reduction factor was less than 1.1 (59). Because population density is strongly influenced by body mass (4), we also performed a phylogenetic generalized linear squares regression of population density on body mass using the package 'caper' in $\mathrm{R}(60)$ and compared the residuals from this regression between socially 510 monogamous and solitary species. 
Supplementary Table S1: Overview of the distribution of social monogamy across mammalian Orders

\begin{tabular}{|c|c|c|c|}
\hline Order & $\begin{array}{l}\text { Species } \\
\text { classified }\end{array}$ & $\begin{array}{l}\text { Species with social } \\
\text { monogamy }\end{array}$ & $\begin{array}{l}\text { Proportion of socially } \\
\text { monogamous species }\end{array}$ \\
\hline AFROSORICIDA & 11 & & $0 \%$ \\
\hline ARTIODACTYLA & 187 & 6 & $3 \%$ \\
\hline CARNIVORA & 202 & 32 & $16 \%$ \\
\hline CETACEA & 54 & & $0 \%$ \\
\hline CHIROPTERA & 174 & 10 & $6 \%$ \\
\hline CINGULATA & 14 & & $0 \%$ \\
\hline DASYUROMORPHIA & 14 & & $0 \%$ \\
\hline DIDELPHIMORPHIA & 13 & & $0 \%$ \\
\hline DIPROTODONTIA & 15 & 2 & $13 \%$ \\
\hline ERINACEOMORPHA & 15 & & $0 \%$ \\
\hline HYRACOIDEA & 3 & & $0 \%$ \\
\hline LAGOMORPHA & 72 & 1 & $1 \%$ \\
\hline MACROSCELIDEA & 15 & 14 & $93 \%$ \\
\hline MONOTREMATA & 5 & & $0 \%$ \\
\hline NOTORYCTEMORPHIA & 2 & & $0 \%$ \\
\hline PERAMELEMORPHIA & 5 & & $0 \%$ \\
\hline PERISSODACTYLA & 16 & & $0 \%$ \\
\hline PHOLIDOTA & 8 & & $0 \%$ \\
\hline PILOSA & 10 & & $0 \%$ \\
\hline PRIMATES & 359 & 104 & $29 \%$ \\
\hline PROBOSCIDEA & 3 & & $0 \%$ \\
\hline RODENTIA & 941 & 56 & $6 \%$ \\
\hline SCANDENTIA & 16 & & $0 \%$ \\
\hline SIRENIA & 1 & & $0 \%$ \\
\hline SORICOMORPHA & 384 & 1 & $0 \%$ \\
\hline TUBULIDENTATA & 1 & & $0 \%$ \\
\hline Total & 2543 & 226 & $9 \%$ \\
\hline
\end{tabular}




\section{Supplementary Table S2: Likelihood comparisons of different Bayestraits' Discrete models}

We first compared models assuming a dependent evolution between the two traits under analysis to models assuming an independent evolution. If a model of dependent evolution had a significantly better likelihood, we constructed dependent models that constrained the

520 relevant transition rate (e.g. from solitary ancestors to social monogamy). Because models investigating the evolution of social monogamy from solitary ancestors that included all species did not converge, we run separate analyses for each of the Orders which contain more than 10 socially monogamous species and more than 10 solitary species. In all instances where the best model assumes a dependent evolution, the transition rate to social monogamy from an ancestor that is not solitary is estimated to be zero.

\begin{tabular}{|c|c|c|c|c|}
\hline & & $\begin{aligned} & \text { Log-I } \\
& \text { th }\end{aligned}$ & $\begin{array}{l}\text { nood of a model a } \\
\text { olution of the trait }\end{array}$ & uming \\
\hline & & independent & $\begin{array}{l}\text { dependent and } \\
\text { unconstrained }\end{array}$ & $\begin{array}{l}\text { dependent and } \\
\text { constrained }\end{array}$ \\
\hline $\begin{array}{l}\text { Evolution of social monogamy from } \\
\text { solitary ancestors }\end{array}$ & $\begin{array}{c}\text { Primates } \\
\text { Carnivores } \\
\text { Rodents } \\
\text { Chiroptera }\end{array}$ & $\begin{array}{r}-63.46 \\
-99.37 \\
-308.45 \\
-111.14\end{array}$ & $\begin{array}{r}-54.57 \\
-94.92 \\
-303.45 \\
-101.10\end{array}$ & $\begin{array}{r}-59.07 \\
-106.51 \\
-312.95 \\
-113.72\end{array}$ \\
\hline $\begin{array}{c}\text { Evolution of paternal care in socially } \\
\text { monogamous ancestors }\end{array}$ & & -408.23 & -384.58 & -388.72 \\
\hline $\begin{array}{l}\text { Evolution of social monogamy and } \\
\text { male infanticide }\end{array}$ & & -123.93 & -122.43 & \\
\hline $\begin{array}{l}\text { Evolution of social monogamy and } \\
\text { infanticide risk }\end{array}$ & & -526.46 & -524.4 & \\
\hline
\end{tabular}

